\title{
Calidad percibida e intenciones futuras en eventos deportivos: segmentación de participantes de carreras por montaña
}

\author{
Perceived quality and future intentions in sport events: segmentation \\ of participants of trail running \\ Miguel Madruga Vicente ${ }^{1}$, David Cerro Herrero', Salvador Angosto Sánchez ${ }^{2}$, Josué Prieto Prieto ${ }^{3}$ \\ 1 Facultad de Ciencias del Deporte. Universidad de Extremadura. España. \\ 2 Facultad de Ciencias del Deporte. Universidad de Murcia. España. \\ 3 Escuela Universitaria de Educación y Turismo de Ávila. Universidad de Salamanca. España.
}

\section{CORRESPONDENCIA:}

Miguel Madruga Vicente

miguelmadruga@unex.es

Recepción: septiembre 2020 • Aceptación: marzo 2021
CÓMO CITAR EL ARTÍCULO:

Madruga-Vicente, M., Cerro-Herrero, D., Angosto-Sánchez., S., \& Prieto-Prieto, J. (2021). Calidad percibida e intenciones futuras en eventos deportivos: segmentación de participantes de carreras por montaña. Cultura, Ciencia y Deporte, 16(50), 605-615. http://dx.doi.org/10.12800/ccd.v16i50.1584

\section{Resumen}

El incremento de las carreras de trail running genera un enorme interés por conocer el perfil de los participantes, así como la calidad de estos eventos. Los objetivos del estudio eran analizar el perfil y la calidad percibida por parte de los corredores de trail running e identificar los factores socio-deportivos que determinan la calidad del evento. Participaron 366 corredores, 85\% hombres y $15 \%$ mujeres, con una edad media de $39.40 \pm 8.1$ años. Se utilizó un cuestionario para la recogida de datos con tres secciones: datos socio-deportivos, calidad percibida y calidad general, así como un análisis de clúster. El perfil del participante es de un corredor de entre 36 y 45 años, con estudios universitarios, con experiencia previa y que entrena tres o más días a la semana. Los participantes muestran altos niveles de calidad percibida, que está determinada por el nivel educativo, el nivel de entrenamiento y los años de experiencia. Además, fueron mayoritarios los participantes que mostraban altas intenciones de volver al evento tras el análisis de clúster realizado. Conocer los perfiles de participantes y evaluar la calidad de un evento es una estrategia útil y necesaria para orientar el diseño de los eventos deportivos, proporcionando futuras mejoras en la organización y en los servicios ofrecidos.

Palabras clave: calidad, corredores, disposición, carreras, trail.

\section{Abstract}

The increase in trail running races has generated a great interest in knowing either the profile of the participants or the perception of the quality of these events. This study aimed to evaluate the profile and the perceived quality of trail runners, and to identify the socio-sport factors that determine the quality of the event. 366 trail runners participated in the study, $85 \%$ men and $15 \%$ women, with an average age of $39.40 \pm 8.1$ years. A questionnaire divided three sections was used for data collection: socio-sports data, perceived quality and general quality, as well as a cluster analysis. The participants' is a runner between 36 and 45 years old, with university studies, with previous experience and who trains three or more days a week. The participants show high levels of perceived quality and this quality is determined by the level of education, the level of training and the years of experience in these races. In addition, the majority of participants showed high intentions of returning to the event after a cluster analysis performed. Knowing the participants' profile and evaluating the quality of events is a useful and necessary strategy to guide the appropriate design of sports events, providing future improvements in the organization and services offered.

Key words: quality, trail runners, willingness, races, trail. 


\section{Introducción}

Las actividades deportivas en entornos naturales han experimentado un crecimiento en la última década (Clawson \& Knetsch, 2013; González \& Mundina 2014).

Esta tendencia creciente de la práctica deportiva al aire libre se pone de manifiesto en los últimos datos publicados en el anuario de estadísticas deportivas de la población española en 2020. Tomando como base este documento, las actividades de senderismo y montañismo representan el 65.9\% del turismo deportivo nacional en 2019 para realizar actividad deportiva, y un $31.8 \%$ en el caso del turista internacional que se desplaza a España por motivos de práctica deportiva (Ministerio de Cultura y Deporte 2020).

Entre estas actividades, cabe destacar el gran crecimiento que han experimentado en los últimos años las carreras por montaña o trail running, tanto en el número de eventos como en participantes a escala nacional (Bataller et al. 2014; Seguí \& Farias 2018) e internacional (Scheer et al. 2020). En Estados Unidos la participación en trail running aumentó de 8.58 millones de corredores en 2016 a 9.15 millones en 2017 (Statista 2020a), duplicándose en el caso de la participación de adultos jóvenes, pasando de 1.02 a 2.05 millones (Statista 2020b). Como consecuencia de ello se ha producido un claro incremento de pruebas deportivas de esta modalidad. En Europa, Cernaianu y Sobry (2015) analizaron los eventos incluidos en la International Trail Running Association (ITRA), encontrando un incremento desde 2003 a 2015 de un evento a más de 350 al año.

En España, Babí et al. (2018) destacan un aumento de un $1000 \%$ en el número de carreras por montaña entre 2008 y 2015 en el conjunto del territorio nacional. Este crecimiento también se ha producido en los diferentes territorios regionales. En un informe elaborado por Ropits Social Running (2016) se destaca que en Cataluña la evolución en el número de eventos ha sido de 38 carreras realizadas en 2008 freente a un total de 447 pruebas en el año 2015, mientras en la Comunidad de Madrid se incrementó de una prueba en 2012 a ocho en el año 2015 (Oñorbe 2015). En Alicante pasaron de 4 pruebas en 2008 a 28 en 2014 (Jiménez-Rubio 2015), y en Extremadura se pasó de 3 carreras en el año 2008 a un total de 23 en 2019 (Federación Extremeña de Montaña y Escalada 2020). En relación al perfil del corredor de trail en España, este se caracteriza por un predominio de hombres con edad comprendida entre 36 y 45 años, estudios superiores, varios años de experiencia previa y niveles considerables de entrenamiento a la semana (Bataller et al. 2014; González-Lázaro et al. 2020; Guíu \& Leyton 2019; Moreno 2014).

La participación en eventos deportivos se ha convertido en una de las actividades de ocio más populares de la población en los últimos años (Kennedy et al. 2019; Theodorakis et al. 2015). Esta globalización y diversificación de los eventos queda reflejada en un crecimiento de la literatura científica relacionada con la temática, evaluando el impacto económico (Barajas et al. 2016), social (Añó, et al. 2012; Hautbois et al. 2020), turístico (González-García, et al. 2018; Mur et al. 2020) o la calidad percibida del evento (Angosto et al. 2016a; Cerro 2018). También se han desarrollado instrumentos de evaluación específicos (Angosto et al. 2016a; Calabuig et al. 2016) para las diferente tipologías de eventos, como los trail running, eventos de pequeña o mediana escala que suelen celebrarse en territorios no muy extensos. Estos eventos generan diversos impactos, positivos o negativos, que suelen ser relevantes en la comunidad (Parra-Camacho et al. 2014; Agorreta et al. 2020; Vergara-Ferri et al. 2020), debiendo ser considerados como una estrategia viable y sostenible en la promoción turística y el desarrollo local de este tipo de territorios (Duglio \& Beltramo, 2017; Valente, 2020).

Ante la amplia oferta y competencia en torno los eventos deportivos, la calidad se ha convertido en un factor clave; algunos elementos como la accesibilidad, los espacios o el ambiente tienen una estrecha relación con el valor percibido, la satisfacción o las intenciones futuras (Biscaia et al. 2017; Moon et al. 2013; Tzetzis et al. 2014) y, por tanto, el análisis de la calidad puede contribuir a la mejora de los eventos desde el punto de vista de la organización y a mejorar la oferta existente para ser un sector más atractivo para los participantes.

En el trail running, la ITRA determina la calidad de los eventos en torno a tres dimensiones de la organización: i) previa a la competición (disponibilidad y calidad de la información, comunicación entre participantes y organización, precio de inscripción, eficiencia de los servicio y actividades extras); ii) durante la competición (puntos de ayuda para participantes, señalización del recorrido, ambientación y servicios para acompañantes); y iii) después de la competición (organización de la línea de meta, servicios médicos, logística, disponibilidad de resultados y respeto por el medio ambiente) (Urbánski 2018). Evaluar la calidad de los eventos exige a los organizadores ser sensibles a las opiniones de los participantes con el propósito de conocer ciertos elementos percibidos por los usuarios, que pueden ser relevantes para la mejora de la calidad en general, y así elaborar estrategias adecuadas para 
una gestión exitosa de los eventos deportivos a partir de los resultados (Angosto et al. 2016a). En general, la calidad percibida del servicio, en un evento deportivo, puede definirse como "la diferencia entre lo que se espera del servicio y lo que un consumidor percibe o recibe del mismo" (Shonk \& Chelladurai 2008, p. 588). Este concepto se relaciona estrechamente con otros constructos, como el valor percibido, la satisfacción o las intenciones futuras (Alguacil et al. 2016; Hyun \& Jordan, 2020; Sato et al. 2014). Estos factores suscitan el interés de los organizadores para mejorar su gestión del evento.

Estudios previos han evidenciado que los factores sociodemográficos de los participantes de eventos deportivos pueden determinar el nivel de calidad percibida (Angosto et al. 2016b; Calabuig et al. 2010). Por ello, conocer los rasgos principales de sus participantes genera un interés en los organizadores, ya que permite orientar su gestión a los diferentes perfiles resultantes de su segmentación (Martínez-Cevallos et al. 2020; Rejón et al. 2017). En este sentido, se ha mostrado que el análisis de segmentación puede conducir a un aumento de la participación en los eventos deportivos (Tkaczynski \& Rundle-Thiele, 2020). Las intenciones futuras pueden ser un elemento clave en la segmentación para determinar los perfiles de participantes, brindando a los organizadores información relevante para personalizar la oferta a su público objetivo. Los estudios de segmentación en gestión deportiva son abundantes, sin embargo, en el ámbito de eventos deportivos no son tan habituales. La literatura existente se ha centrado en identificar grupos de participantes según su motivación para la práctica deportiva (Ogles \& Masters, 2003; Ruiz-Juan \& Zarauz, 2014; Parra-Camacho, et al. 2019; Rejón, et al. 2017). En el contexto de eventos deportivos se encuentra un estudio de segmentación en maratón, identificándose dos grupos de participantes diferenciados en relación a las variables de calidad percibida, satisfacción, valor percibido e intenciones futuras (Martínez-Cevallos, et al. 2020).

Actualmente no existen estudios previos que realicen un análisis de segmentación en las variables de en participantes de carreras de trail running. El objetivo de este estudio es analizar la calidad percibida de los participantes de un circuito de carreras de trail running y la segmentación de perfiles en función de las intenciones futuras, identificando los grupos de participantes según su interés por recomendar o repetir la experiencia en el evento. Además, se comparará el perfil de los grupos de participantes para determinar sus características sociodemográficas y hábitos deportivos.

\section{Método}

\section{Muestra}

La población objeto de estudio está constituida por el total de participantes del Circuito Extremeño de Carreras por Montaña de la Federación Extremeña de Montaña y Escalada. Un total de 3647 corredores asistieron en el conjunto de los 15 eventos que formaban el circuito de pruebas; a través de un muestreo no probabilístico por conveniencia se calculó la muestra mínima, siendo esta de 348 sujetos, determinada para un margen de error del $5 \%$.

368 participantes respondieron al cuestionario, de los cuales 2 quedaron anulados por respuestas inadecuadas o valores erróneos. La muestra final fue de 366 participantes, 312 hombres (85\%) y 54 mujeres (15\%), representando la proporción aproximada de participantes según género, con una edad media de 39.40 $\$ 8.1$ años. Un $63.1 \%$ tenía formación universitaria y un $86.9 \%$ se encontraba trabajando. Respecto a la procedencia de los participantes, el $43.1 \%$ provenía de la provincia de Badajoz, seguido de aquellos que procedían de la provincia de Cáceres (31.9\%).

Los aspectos deportivos mostraron que un $59.0 \%$ no tenía licencia deportiva, el $45.9 \%$ tenía una experiencia en este tipo de pruebas deportivas entre uno y tres años, seguido de aquellos que tenían entre cuatro y seis años de experiencia con un $27.3 \%$. Finalmente, aproximadamente la mitad de los participantes entrenaba entre tres y seis horas semanales (53.0\%).

\section{Instrumento}

El instrumento de recogida de datos fue un cuestionario que estuvo formado por tres secciones. La primera sección eran los datos sociodemográficos y hábitos deportivos del participante a través de ocho ítems (género, edad, nivel de estudios, estado civil, ocupación, procedencia, experiencia deportiva, licencia federativa). La segunda sección estuvo compuesta por el cuestionario de calidad de la calidad percibida del participante en eventos populares (CAPPEP) de Angosto et al. (2016a). El cuestionario quedó integrado por 16 ítems divididos en cuatro factores: i) comunicación, compuesto por tres ítems ( $\alpha$ de Cronbach: 0.684; $\omega$ de McDonald: 0.714 ); ii) trato personal, formado por cuatro ítems ( $\alpha$ de Cronbach: 0.743; $\omega$ de McDonald: 0.765); iii) infraestructura logística con cinco ítems (a de Cronbach: 0.798; $\omega$ de McDonald: 0.805); y iv) servicios complementarios, formado por cuatro ítems ( $\alpha$ de Cronbach: 0.734; $\omega$ de McDonald: 0.744). La fiabilidad de la escala total tuvo un valor de a de Cronbach de 0.891 y una $\omega$ de McDo- 
nald de 0.900. La tercera sección se compuso de seis ítems que valoraron el servicio del evento deportivo en general, distribuyéndose en tres factores: v) dos ítems sobre calidad general del servicio, tomados de Hightower et al. (2002; a de Cronbach: 0.770; $\omega$ de McDonald: 0.791); vi) dos ítems sobre valor percibido, también tomados de Hightower et al. (2002; a de Cronbach: 0.957; $\omega$ de McDonald: 0.957); y vii) dos ítems sobre intenciones futuras, adaptados de Zeithmal et al. (1996; a de Cronbach: 0.925; $\omega$ de McDonald: 0.926). La escala de respuesta fue tipo Likert de cinco puntos de anclaje; en el instrumento se empleó una escala de cinco puntos ( 1 = máximo desacuerdo y 5 = máximo acuerdo).

\section{Procedimiento de recogida de información}

Previamente a la recogida de datos, se contactó con los organizadores de los eventos deportivos pertenecientes al circuito con el objetivo de informar sobre el estudio y conseguir el consentimiento informado para participar. La recogida de datos se realizó mediante un cuestionario autoadministrado vía email con el enlace al instrumento adjunto. Se elaboró utilizando la herramienta Google Formularios, seleccionada de acuerdo a criterios de funcionalidad y operatividad para los participantes. Un envío inicial fue realizado el día después de la celebración de la prueba, y un segundo envío de recordatorio a los cinco días, siendo el plazo de respuesta total de diez días desde el primer envío. La tasa de respuesta fue del 11.9\%, en un rango del 7.5-15.0\%. El cuestionario se realizó de forma voluntaria y anónima, informando previamente de su consentimiento de forma expresa.

\section{Análisis de datos}

El análisis estadístico se realizó con el programa SPSS v24.0 (IBM, Armonk, Nueva York). Se calcularon estadísticos descriptivos para variables continuas y variables cualitativas. También se realizó un análisis de correlación de Pearson para observar la relación entre factores. A continuación, se llevó a cabo un análisis de conglomerados para identificar posibles grupos de participantes tomando como variable dependiente la variable intenciones futuras. Para obtener las soluciones de los clústeres se combinaron dos métodos, jerárquicos y no jerárquicos, con el fin de optimizar los resultados. Los análisis de conglomerados se llevaron a cabo utilizando las directrices propuestas por Romesburg (1984). El análisis del conglomerado jerárquico se realizó tomando como referencia el método de Ward para el proceso de agrupación, ya que permite determinar que los clústeres se constituyan de manera que, al unirse dos elementos, la pérdida de la información entre ambos sea mínima (Ward 1963). A su vez este método implica observaciones equiponderadas para evaluar las disimilitudes distintas existentes entre ambos elementos, a través del cuadrado de la distancia euclidiana (Murtagh \& Legendre 2014). Posteriormente se hizo un clúster no jerárquico a través del método de K-means, tomando como referencia los centros de las soluciones de clúster del método jerárquico para cada período. Una vez determinada la solución ideal del cúmulo según los criterios expuestos por Hair et al. (2014), se determinaron los perfiles de los diferentes grupos utilizando todas las variables no incluidas en el análisis de cúmulos. Las pruebas de chi cuadrado, calculando el valor del coeficiente de contingencia $\left(\mathrm{C}^{2}\right)$ para verificar el tamaño del efecto y la intensidad de la asociación entre las variables cualitativas, compararon los resultados mediante la realización de la prueba de ANOVA para las variables continuas y para las cualitativas (Khalilzadeh \& Tasci 2017). La asociación entre las variables estudiadas se ratificó con una regresión lineal considerando como variables dependientes el total de calidad percibida y calidad total y, como variables independientes, los factores socio-deportivos. La fiabilidad de la escala se calculó a través de los índices a de Cronbach y $\omega$ de McDonald (Revelle 2019). El nivel de significación se estableció en un valor de $p \leq 0,05$.

\section{Resultados}

\section{Análisis de correlaciones}

La Tabla 1 muestra el análisis descriptivo y las correlaciones de los factores. Los descriptivos mostraron que el trato personal fue el factor mejor valorado por los participantes, seguido de la calidad general del servicio. Por el contrario, los servicios complementarios y el valor percibido fueron los factores peor valorados. Los resultados indicaron que hubo relaciones positivas y significativas entre todos los factores que compusieron las variables de estudio.

\section{Identificación y descripción de clústeres}

La Tabla 2 muestra los centros de cada grupo de los diferentes ítems de calidad percibida del evento y calidad total del servicio atendiendo a la solución de tres clústeres. Los resultados del test ANOVA confirmaron la existencia de diferencias significativas en todas las variables $(p \leq 0.001)$, siendo más relevantes las encontradas en los factores de calidad general del servicio, valor percibido y servicios complementarios. El 
Tabla 1. Descriptivos y análisis de correlaciones de los factores.

\begin{tabular}{|c|c|c|c|c|c|c|c|c|c|c|}
\hline Factores & $M(D T)$ & 1 & 2 & 3 & 4 & 5 & 6 & 7 & 8 & 9 \\
\hline 1. Comunicación & $4.20(0.7)$ & - & & & & & & & & \\
\hline 2. Trato personal & $4.69(0.4)$ & $0.503 *$ & - & & & & & & & \\
\hline 3. Infraestructura logística & $4.22(0.7)$ & $0.528 *$ & $0.603 *$ & - & & & & & & \\
\hline 4. Servicios complementarios & $4.16(0.7)$ & $0.610 *$ & $0.577^{*}$ & $0.605^{*}$ & - & & & & & \\
\hline 5. Calidad general servicio & $4.40(0.7)$ & $0.596 *$ & $0.590 *$ & $0.585^{*}$ & $0.698 *$ & - & & & & \\
\hline 6. Valor percibido & $4.07(0.8)$ & $0.487^{*}$ & $0.526 *$ & $0.561 *$ & $0.712 *$ & $0.791 *$ & - & & & \\
\hline 7. Intenciones futuras & $4.33(0.8)$ & $0.468 *$ & $0.498 *$ & $0.498^{*}$ & $0.673^{*}$ & $0.844 *$ & $0.855^{*}$ & - & & \\
\hline 8. Total calidad percibida & $4.32(0.5)$ & $0.778^{*}$ & $0.772 *$ & $0.843^{*}$ & $0.885^{\star}$ & $0.753 *$ & $0.711 *$ & $0.663^{*}$ & - & \\
\hline 9. Total valoración calidad & $4.27(0.7)$ & $0.543^{*}$ & $0.568 *$ & $0.579 *$ & $0.737^{*}$ & $0.924 *$ & $0.943^{*}$ & $0.958 *$ & $0.750 *$ & - \\
\hline
\end{tabular}

Nota * $p<0.001$.

Tabla 2. Conglomerados medios de los factores de calidad percibida en los tres grupos clúster.

\begin{tabular}{|c|c|c|c|}
\hline & $\begin{array}{l}\text { Altas intenciones } \\
\qquad(n=237)\end{array}$ & $\begin{array}{l}\text { Intenciones moderadas } \\
\qquad(n=115)\end{array}$ & $\begin{array}{l}\text { Bajas intenciones } \\
\qquad(n=15)\end{array}$ \\
\hline & $M(D T)$ & $M(D T)$ & $M(D T)$ \\
\hline Total Calidad Percibida* $(F(364)=106.62 ; p \leq 0.001)$ & $4.54(0.4)$ & $3.96(0.4)$ & $3.49(0.8)$ \\
\hline Comunicación* $(F(364)=55.20 ; p \leq 0.001)$ & $4.43(0.6)$ & $3.73(0.6)$ & $3.40(0.8)$ \\
\hline Trato Personal* $(F(364)=56.63 ; p \leq 0.001)$ & $4.84(0.3)$ & $4.47(0.4)$ & $4.12(0.9)$ \\
\hline Infraestructura logística* $(F(364)=45.60 ; p \leq 0.001)$ & $4.44(0.6)$ & $3.89(0.7)$ & $3.30(1.1)$ \\
\hline Servicios Complementarios* $(F(364)=91.61 ; p \leq 0.001)$ & $4.44(0.5)$ & $3.70(0.6)$ & $3.19(0.9)$ \\
\hline Total valoración del servicio* $(F(364)=423.78 ; p \leq 0.001)$ & $4.69(0.3)$ & $3.65(0.4)$ & $2.37(0.9)$ \\
\hline Calidad General* $(F(364)=466.83 ; p \leq 0.001)$ & $4.80(0.3)$ & $3.83(0.4)$ & $2.57(0.8)$ \\
\hline Valor Percibido* $(F(364)=152.21 ; p \leq 0.001)$ & $4.47(0.6)$ & $3.47(0.6)$ & $2.43(1.0)$ \\
\hline Intenciones Futuras* $(F(364)=498.76 ; p \leq 0.001)$ & $4.80(0.3)$ & $3.65(0.5)$ & $2.10(0.8)$ \\
\hline
\end{tabular}

Nota * $\mathrm{p} \leq$ 0.001. M = Media; DT = Desviación Estándar; F = Estadístico.

Clúster 1 estuvo formado por un $64.6 \%$ del total de participantes encuestados y se denominó "Altas intenciones" debido a que obtuvieron una muy alta intención futura respecto al evento. Los factores asociados mejor valorados fueron el trato personal, la calidad general del servicio y el valor percibido. El Clúster 2 se compuso de un total de 115 participantes (31.3\%) y se llamó "Intenciones moderadas" debido a puntuaciones neutrales-moderadas en sus intenciones futuras respecto al evento. Los resultados indicaron que el total de la calidad percibida tuvo puntuaciones moderadasaltas en el trato personal, seguido de la infraestructura logística y la calidad general del servicio. Finalmente, el Clúster 3, denominado "Bajas intenciones", fue el menos numeroso (4.1\%) y en el que se mostraron unas intenciones futuras moderadas-bajas. Los resultados de calidad percibida fueron moderados, valorándose positivamente el trato personal, mientras el resto de los factores fueron neutros.

\section{Perfil sociodemográfico de los grupos clúster}

Los perfiles sociodemográficos y deportivos de los participantes según el grupo clúster aparecen en la Tabla 3. Los tres grupos clúster tuvieron un perfil sociodemográfico similar, compuesto principalmente por hombres de entre 36 y 45 años y formación universitaria. Por su parte, el perfil deportivo del Clúster 1 y Clúster 2 mostró que los participantes no tenían licencia deportiva, contaban con poca experiencia previa y no entrenaban mucho tiempo (menos de 6 horas semanales). En cambio, en el Clúster 3 la mayoría de las participantes sí tenía licencia federativa, una mayor experiencia y entrenaba más tiempo. Los resultados comparativos no mostraron diferencias estadísticamente significativas ( $p>0.05$ ). Todas las variables mostraron intensidades en su relación del tamaño del efecto bajas o muy bajas.

\section{Análisis de regresión lineal}

Por último, la Tabla 4 muestra la asociación entre las variables socio-deportivas y las escalas de calidad percibida y calidad general. Los resultados del análisis muestran que el nivel de estudios y la condición de federado determinan de forma significativa $(\mathrm{p}<0.01)$ los niveles de calidad percibida del participante. Del mismo modo, la condición de federado del participante es un factor que determina la valoración sobre la calidad general del evento $(\mathrm{p}<0.01)$. 
Tabla 3. Perfil socio-deportivo de los corredores de trail running según intenciones futuras.

\begin{tabular}{|c|c|c|c|}
\hline \multirow{2}{*}{ Variables } & $\begin{array}{l}\text { Altas intenciones } \\
\quad(n=237)\end{array}$ & $\begin{array}{c}\text { Intenciones moderadas } \\
\quad(n=115)\end{array}$ & $\begin{array}{c}\text { Bajas intenciones } \\
\quad(n=15)\end{array}$ \\
\hline & $N(\%)$ & $N(\%)$ & $N(\%)$ \\
\hline \multicolumn{4}{|c|}{ Género $\left(x^{2}=2.98(2) ; p=0.226 ; C^{2}=0.090\right)$} \\
\hline Hombre & 199(84.0) & $99(86.1 \%)$ & $15(100.0)$ \\
\hline Mujer & $38(16.0)$ & $16(13.9 \%)$ & - \\
\hline \multicolumn{4}{|c|}{$\operatorname{Edad}\left(x^{2}=1.18(4) ; p=0.881 ; C^{2}=0.057\right)$} \\
\hline Entre 18 y 35 años & $72(30.4)$ & $31(27.0)$ & $4(26.7)$ \\
\hline Entre 36 y 45 años & $126(53.2)$ & $61(53.0)$ & $9(60.0)$ \\
\hline De 46 en adelante & $39(16.5)$ & 23(20.0) & $2(13.3)$ \\
\hline \multicolumn{4}{|c|}{ Nivel de estudios $\left(X^{2}=3.26(2) ; p=0.196 ; C^{2}=0.094\right)$} \\
\hline Universitarios & $142(59.9)$ & $79(68.7)$ & $11(73.3)$ \\
\hline No universitarios & $95(40.1)$ & $36(31.3)$ & $4(26.7)$ \\
\hline \multicolumn{4}{|c|}{ Situación laboral $\left(X^{2}=6.27(8) ; p=0.617 ; C^{2}=0.13\right)$} \\
\hline Empleado/a & $210(88.6)$ & $96(83.5)$ & $13(86.7)$ \\
\hline Amo/a de casa & $3(1.3)$ & - & - \\
\hline Desempleado/a & $14(5.9)$ & $9(7.8)$ & $1(6.7)$ \\
\hline Estudiante & $6(2.5)$ & $5(4.3)$ & 1(6.7) \\
\hline Jubilado/pensionista & $4(1.7)$ & $5(4.3)$ & - \\
\hline \multicolumn{4}{|c|}{ Procedencia (provincia) $\left(x^{2}=5.58(4) ; p=0.233 ; C^{2}=0.122\right)$} \\
\hline Cáceres & 74(31.2) & 39(33.9) & $4(26.7)$ \\
\hline Badajoz & $109(46.0)$ & $45(39.1)$ & $4(26.7)$ \\
\hline Otras & $54(22.8)$ & $31(27.0)$ & $7(46.6)$ \\
\hline \multicolumn{4}{|c|}{ Federado (licencia) $\left(x^{2}=4.96(2) ; p=0.084 ; C^{2}=0.116\right)$} \\
\hline Sí & $87(36.7)$ & $55(47.8)$ & $8(53.3)$ \\
\hline No & $150(63.3)$ & $60(52.2)$ & $7(46.7)$ \\
\hline \multicolumn{4}{|c|}{ Experiencia (años) $\left(x^{2}=8.32(8) ; p=0.403 ; c^{2}=0.149\right)$} \\
\hline Menos de 1 año & 28(11.8) & $12(10.4)$ & 1(6.7) \\
\hline De 1 a 3 años & $112(47.3)$ & $54(47.0)$ & $3(20.0)$ \\
\hline De 4 a 6 años & $61(25.7)$ & $31(27.0)$ & $8(53.3)$ \\
\hline De 6 a 10 años & 26(11.0) & 13(11.3) & $3(20.0)$ \\
\hline Más de 10 años & $10(4.2)$ & $5(4.3)$ & - \\
\hline \multicolumn{4}{|c|}{ Entrenamiento (horas/semana) $\left(x^{2}=11.7(6) ; p=0.069 ; C^{2}=0.176\right)$} \\
\hline Entre 0 y 3 horas & 19(8.0) & 20(17.4) & 1(6.7) \\
\hline Entre 3 y 6 horas & $129(54.4)$ & $60(52.2)$ & $5(33.3)$ \\
\hline Entre 6 y 10 horas & 75(31.6) & $31(27.0)$ & $8(53.3)$ \\
\hline Más de 10 horas & $14(5.9)$ & $4(3.5)$ & $1(6.7)$ \\
\hline
\end{tabular}

Nota: $x^{2}=$ Chi-cuadrado Pearson; $C 2=$ Coeficiente de contingencia.

Tabla 4. Regresión lineal para analizar las relaciones de las escalas de calidad percibida y calidad general con las variables wsocio-deportivas del participante.

\begin{tabular}{|c|c|c|c|c|c|c|}
\hline & \multicolumn{3}{|c|}{ Modelo Calidad Percibida } & \multicolumn{3}{|c|}{ Modelo Calidad General } \\
\hline & B estandarizado & $\mathbf{t}$ & $p$ & B estandarizado & $t$ & $p$ \\
\hline Constante & & 19.500 & $.000 * *$ & & 12.028 & $.000 * *$ \\
\hline Sexo & .023 & .440 & .660 & .085 & 1.609 & .108 \\
\hline Grupo Edad & .036 & .660 & .510 & .001 & 0.16 & .987 \\
\hline Nivel Estudios & .150 & 2.876 & $.004 * *$ & .108 & 2.050 & .041 \\
\hline Condición Federado & .164 & 3.182 & $.002 * *$ & .143 & 2.736 & $.007 * *$ \\
\hline Años Experiencia & -.080 & -1.384 & .167 & -.064 & -1.091 & .276 \\
\hline Horas Entrenamiento & -.060 & -1.101 & .272 & .053 & .953 & .341 \\
\hline
\end{tabular}

Nota * $p<0.05 ; * * p<0.01$ 


\section{Discusión}

El objetivo de este estudio fue analizar la calidad percibida por los corredores que participaron en el circuito extremeño de carreras de trail running y analizar los perfiles socio-deportivos de los participantes, observando sus posibles diferencias en la valoración del evento. Los resultados mostraron que los factores de la calidad del evento se relacionaron significativamente entre ellos, por lo que la calidad de los eventos es un elemento que depende de la combinación de procesos internos (satisfacción, sensaciones, percepciones) y externos (características eventos, servicios ofrecidos, etc.) (Calabuig et al., 2012). Como se ha comentado anteriormente, la segmentación dentro del sector de eventos deportivos ha sido amplia, ya que conocer los perfiles específicos de participantes permite elaborar mejores campañas de marketing enfocadas a un perfil específico. El análisis de los grupos mostró que la mayor proporción de los participantes encuestados se agruparon en el Clúster 1, "Altas intenciones", mostrando elevados valores en la calidad percibida y calidad total. El Clúster 2, "Intenciones moderadas", mostró puntuaciones moderadas-altas en la mayoría de los factores, mientras el Clúster 3, "Bajas intenciones", fue el menos numeroso y tuvo valoraciones moderadas en los factores de calidad percibida y bajas en la calidad total. Por tanto, las expectativas de los participantes se han visto generalmente cubiertas (Angosto et al., 2016b), de modo que las intenciones son indicadores útiles para el organizador, dado que le proporcionan información sobre la calidad y pueden promover que el participante vuelva a asistir en sucesivas ediciones.

Atendiendo a los factores específicos, el trato personal fue el factor de calidad percibida con puntuaciones más altas en los tres grupos, obteniendo mayor puntuación en la calidad general entre los factores de calidad total. La literatura indica que la prestación de un servicio de alta calidad genera efectos positivos sobre los participantes, mejorando sus intenciones de realizar ejercicio regular (Chen et al. 2012; Ma \& Kaplanidou 2018). El factor personal suele ser el más determinante dentro de la calidad del servicio (Bodet 2006), encontrándose el trato personal como la dimensión mejor valorada en estudios previos (Alexandris et al. 2017; Angosto et al., 2016a, 2016b; Calabuig et al. 2010; Kim et al. 2013; Theodorakis et al. 2015). Los factores inherentes al desarrollo del evento también influyen en una alta percepción de calidad (Angosto et al. 2016a; González 2019; Moreno 2014). A su vez, los factores peor valorados de cada escala, servicios complementarios y valor percibido, coincidieron con los estudios de Angosto et al. (2016a, 2016b).
Estudios previos mostraron que los participantes de eventos deportivos en general manifiestan niveles de satisfacción elevados (Benjamim 2019; González 2019). Del mismo modo, de acuerdo con Moreno (2014) y Theodorakis et al. (2015), los participantes en pruebas de trail running manifiestan también altos niveles de satisfacción, ya que este tipo de eventos puede proporcionarles sentimientos de placer y afectos positivos durante un tiempo (Sato et al. 2014). Además, ese sentimiento de satisfacción en los participantes de estas pruebas puede verse favorecido por la superación de estos retos, y los sentimientos de felicidad que les producen, lo cual podría contribuir a mejorar su satisfacción personal con el evento (Guíu \& Leyton 2019; Theodorakis et al. 2015). Sin embargo, Alexandris et al. (2017) establecieron que el nivel de participación en un evento no es solo consecuencia de la calidad del servicio, sino que también influyen otros factores en la percepción de calidad del evento, como el nivel deportivo de los participantes que asisten, por lo que eventos más icónicos pueden atraer más participantes. A su vez, Ahrholdt et al. (2017) argumentaron que la percepción de calidad influirá según la experiencia de los participantes, demostrándose en este estudio que el Clúster 1, "Altas intenciones", y el Clúster 2, "Moderadas intenciones", fueron los grupos donde mayor proporción de participantes había en las categorías con menor tiempo de experiencia en este tipo de pruebas, mientras el Clúster 3 tuvo mayores proporciones en las categorías de mayor experiencia.

Además de los factores propios del evento, existen otros factores relacionados con el perfil del participante que pueden influir en la valoración de la calidad. Como destacan Roca-Cruz et al. (2019), la calidad percibida de los participantes de un evento deportivo va más allá de los aspectos tangibles, personal que asiste a los deportistas, accesibilidad al lugar y servicios complementarios ofrecidos, por lo que características propias del corredor, como el nivel educativo o el estar federado, también podrían modular su percepción de la calidad.

En relación al perfil sociodemográfico y deportivo, los resultados mostraron que el perfil del participante en las pruebas de trail corresponde a un corredor varón de entre 36 y 45 años, con estudios universitarios, con varios años de experiencia previa y que entrena tres o más días a la semana. Las características descritas están en concordancia con estudios previos (Alguacil et al. 2018; Angosto et al. 2016a, 2016b; González-Lázaro et al. 2020; Guíu \& Leyton 2019; Moreno 2014). Sin embargo, en carreras populares los participantes tenían edades inferiores, en torno a los 30 años (Prieto 2016; González 2019). En lo refe- 
rido al nivel de estudios, los resultados de este estudio están en acuerdo con González (2019) y Babí (2018), determinando que la mayoría de los participantes posee estudios universitarios, siendo mayor el porcentaje en las mujeres. En España, Bataller et al. (2014) compararon el perfil más habitual en participantes de carreras populares y trail running, encontrando que el corredor de trail running suele tener en mayor proporción licencia federativa que el corredor popular, siendo el perfil más habitual el de personas de entre 36 y 50 años, que vive en pareja, con hijos, reside en el ámbito urbano, pertenece a la clase media-alta y prefiere la práctica en solitario y sin entrenador, habiendo practicado anteriormente otras modalidades deportivas. Otros estudios más recientes establecen que el perfil de los participantes de pruebas de trail running es similar al de otras pruebas populares: hombre, de edad comprendida entre 36 y 45 años, con estudios superiores, con años de experiencia previa y niveles considerables de entrenamiento a la semana (González-Lázaro et al. 2020; Guíu \& Leyton 2019; Moreno 2014). Por otra parte, los resultados confirman también que los participantes de las pruebas de trail running acumulan varios años de experiencia previa y de entrenamiento en la modalidad, si bien existen diferencias en función del género, de manera que los hombres acumulan más tiempo de experiencia que las mujeres (Alguacil et al. 2018; Babí et al. 2018; Guíu \& Leyton 2019).

\section{Implicaciones y limitaciones del estudio}

Hasta donde alcanza nuestro conocimiento no existen estudios específicos en España que analicen la calidad percibida en los participantes de pruebas de trail running. Desde el punto de vista de la aplicabilidad del estudio, los resultados de esta evaluación permitirían a los organizadores conocer las necesidades de sus clientes para mejorar la calidad del servicio (Cerro 2018). Del mismo modo, facilitarían al organizador orientar la atención hacia un determinado perfil de usuario, así como facilitar la evaluación de los potenciales participantes en futuros eventos deportivos y mejorar las estrategias de planificación, desarrollo y ejecución de los eventos por parte de los organizadores (Angosto et al. 2016a; Cerro 2018). Finalmente, posibilitaría que el organizador pudiera añadir, si fuera necesario, medidas de mejora para favorecer que este tipo de pruebas sean sostenibles y sustentables temporal y territorialmente en todos los ámbitos (Seguí \& Farías 2018). No obstante, para incrementar la sostenibilidad en el tiempo y sobre todo el impac- to social, económico y de desarrollo de estos eventos en las zonas donde tienen lugar (generalmente zonas rurales), es necesario añadir otros factores que contribuyan al desarrollo de la actividad turística, siendo la percepción de los residentes y de las entidades locales (empresas, asociaciones, ayuntamiento, etc.) algunos de los factores más relevantes (González-García et al. 2019; Karadakis 2012). Del mismo modo, conocer el perfil y la procedencia de los participantes, así como sus intenciones futuras, podría facilitar a los organizadores la gestión de dichos eventos, principalmente en la planificación de estrategias de captación y difusión en otras zonas más alejadas de su entorno, donde se ha demostrado que las intenciones son más bajas. Para ello se requieren futuros estudios que traten de analizar estos dos objetivos, evaluar los aspectos relacionados con el impacto ambiental y sostenibilidad de estos eventos y los aspectos relacionados con la mejora de las estrategias de difusión y planificación.

El presente estudio presenta algunas limitaciones. En primer lugar, el muestreo no probabilístico por conveniencia impide la generalización de los resultados obtenidos. Por otro lado, el tamaño de la muestra comprende diferentes pruebas deportivas con un número variable de respuesta en cada una, debiendo tratar los datos con cautela, aunque los perfiles y características son coherentes con estudios previos. Futuros estudios deberían contemplar la realización de un muestreo adecuado en las diferentes pruebas de las que consta el circuito para poder generalizar los resultados del trail running en Extremadura. Por otro lado, sería interesante poder realizar una evaluación longitudinal del mismo sujeto en las diferentes pruebas o poder realizar estudios similares en otras comunidades y comparar la evaluación de la calidad percibida del evento y los perfiles sociodemográficos de la población. Por último, futuros estudios deberían incidir en la evaluación de la percepción medioambiental de este tipo de pruebas por parte del participante y el nivel de sostenibilidad del evento.

\section{Conclusiones}

La evaluación de los participantes de pruebas de trail running nos ha permitido identificar 3 perfiles en función de las intenciones futuras, de manera que la mayoría de ellos muestra altas intenciones de volver al evento.

La evaluación de la calidad de un evento deportivo está determinada por multitud de factores, algunos de ellos relacionados con el participante, como son los factores socio-deportivos (el nivel de estudios y la 
condición de federado). Conocer en profundidad estos factores puede favorecer la gestión al organizador a la hora de desarrollar un evento deportivo y maximizar en la medida de lo posible todos los elementos de calidad de este, así como poder adaptarlos a los distintos perfiles de los participantes.

Los elevados niveles de calidad general y percibida en los eventos deportivos en Extremadura favorecen que los participantes manifiesten un importante deseo de volver a competir en las pruebas. Por ello, los participantes pueden jugar un papel muy importante a la hora de promover los eventos en sucesivos años, ya que pueden actuar como prescriptores y embajadores del evento y de la localidad donde se desarrollan si la experiencia es satisfactoria.

Por último, la aplicación de herramientas de evaluación de la calidad del evento desde la perspectiva del participante es una estrategia útil y necesaria que permite a los organizadores conocer los intereses de los participantes, de modo que pueda orientar el diseño de los eventos deportivos en el futuro, proporcionando mejoras en la organización y la gestión que permitan incrementar la eficiencia y el impacto que genere en la zona donde se desarrollan. Este tipo de estudios permite hacer más participes de la organización del evento a los corredores, mejorando así su fidelidad.

\section{BIBLIOGRAFÍA}

Agorreta, J., Madruga, M., Cerro, D. , \& Prieto, J. (2020). Impacto socioeconómico del turismo activo de eventos deportivos: Un estudio exploratorio para el caso del Valle del Jerte. Rotur: revista de ocio y turismo, 14(2), 88-104. https://doi.org/10.17979/rotur.2020.14.2.6436

Ahrholdt, D. C., Gudergan, S. P., \& Ringle, C. M. (2017). Enhancing service loyalty: The roles of delight, satisfaction, and service quality. Journal of Travel Research, 56(4), 436-450. https://doi. org/10.1177/0047287516649058.

Alexandris, K., Theodorakis, N., Kaplanidou, K., \& Papadimitriou, D. (2017). Event quality and loyalty among runners with different running involvement levels. International Journal of Event and Festival Management, 8(3), 292-307. https://doi.org/10.1108/IJEFM-08-2016-0057.

Alguacil, M., Parra-Camacho, D., \& Giménez-Espert, C. (2018). Características de los participantes en el Circuito de Carreras Populares de la ciudad de Valencia. Calidad de Vida y Salud, 11(7); 37-47. https://doi. org/10.6018/254131.

Alguacil, M., Perez-Campos, C., Berenguer, S. A., \& Boquera, J. (2016). Does hosting a sport event promotes the city among athletes as a tourist destination?. Sport Tk-Revista Euroamericana de Ciencias del Deporte, 5(1), 123-136. https://doi.org/10.6018/254131

Angosto, S., López-Gullón, J. M., \& Díaz, A. (2016a). Una escala para la evaluación de la calidad percibida por participantes en eventos deportivos populares (CAPPEP V2. 0). Journal of Sports Economics \& Management, 6(2), 69-84. http://sportsem.uv.es/j_sports_and_em/index. $\mathrm{php} / \mathrm{JSEM} /$ article/download/50/74

Angosto, S., López-Gullón, J. M., \& Díaz, A. (2016b). Participants' perceived quality in two editions of a popular race. Intangible Capital, 12(3), 789-804. http://dx.doi.org/10.3926/ic.782.

Añó, V., \& Calabuig, F., \& Parra, D. (2012). Impacto social de un gran evento deportivo: el Gran Premio de Europa de Fórmula 1. Cultura, Ciencia y Deporte, 7(19),53-6.

Babí, J., Inglés, E., Cumellas, L., Farías, E., Seguí, J., \& Labrador, V. (2018). El perfil de los corredores y su propensión al accidente deportivo. Revista Internacional de Medicina y Ciencias de La Actividad Física y Del Deporte, 18(72), 737-752. https://doi.org/10.15366/rimcafd2018.72.009.

Barajas, A., Coates, D., \& Sánchez-Fernández, P. (2016) Beyond retrospective assessment. Sport event economic impact studies as a management tool for informing event organization. European Research on Management and Business Economics, 22(3), 124-130. https://doi. org/10.1016/j.iedee.2015.05.001

Bataller, V., Marcén, C., Piedrafita, E., \& Arbonés, I. (2014). Comparación de los perfiles de corredores de carreras populares y corredores de carreras por montaña. En R. LLopis (dir.). Crisis, cambio social y deporte. XIII Congreso Internacional de la Asociación Española de Investigación Social Aplicada al Deporte (AEISAD). Investigación Social y Deporte, 12 (97-104). Nau Llibres.
Benjamim, V. (2019). Organização e gestão de eventos desportivos: a satisfação dos participantes da copa up. Zona sul, maxixe-2016. Revista Intercontinental de Gestão Desportiva, 9(3), 16-28. http://www.revista. universo.edu.br/index.php?journal=gestaoesportiva\&page=article\& op=view\&path\%5B\%5D=7820\&path\%5B\%5D=3976

Biscaia, R., Correia, A., Santos, T., Ross, S., \& Yoshida, M. (2017). Service quality and value perceptions of the 2014 FIFA World Cup in Brazil. Event Management, 21(2), 201-216. https://doi.org/10.3727/152 $599517 X 14878772869685$.

Bodet, G. (2006). Investigating customer satisfaction in a health club context by an application of the tetraclasse model. European Sport Management Quarterly, 6(2), 149-165. https://doi.org/10.1080/1618474 0600954148.

Calabuig, F., Burillo, P., Crespo, J., Mundina, J. J., \& Gallardo, L. (2010). Satisfaction, quality and perceived value in spectators of athletics. Revista Internacional de Medicina y Ciencias de la Actividad Física y el Deporte, 10(40), 577-593. http://cdeporte.rediris.es/revista/revista40/ artsatisfaccion182.htm.

Calabuig, F., Crespo-Hervas, J., Prado-Gasco, V., Mundina, J., Valantine, I., \& Stanislovaitis, A. (2016). Quality of sporting events: Validation of the EVENTQUAL scale. Transformations in Business \& Economics,15(2), 21-32. http://hdl.handle.net/11268/7423

Calabuig, F., Molina, N., \& Núñez, J. (2012). Una aplicación inicial del modelo tridimensional de calidad de servicio en centros deportivos privados. E-Balonmano: Revista de Ciencias del Deporte, 8(1), 67-81.

Cernaianu, S., \& Sobry, C. (2015). Evolution and impact on the environment of trail running sporting Events-the case of France and Romania. En M. Plevnik, I. Retar, R. Pisot \& A, Obid. Sustainable Development of Sports Tourism ( $\mathrm{p} 81$ ). Annales University Press.

Cerro, D. (2018). Análisis de la calidad percibida y satisfacción de participantes en eventos deportivos. En Universidad de Extremadura (Ed.), Catálogo de investigación joven en Extremadura: Volumen I (pp. 66-70).

Chen, L. H., Chen, M. Y., Ye, Y. C., Tung, I. W., Cheng, C. F., \& Tung, S. (2012). Perceived service quality and life satisfaction: the mediating role of the actor's satisfaction-with-event. International Journal of Sports Marketing and Sponsorship, 13(4), 7-24. https://doi. org/10.1108/IJSMS-13-04-2012-B003.

Clawson, M., \& Knetsch, J. L. (2013). Economics of outdoor recreation (vol 3). RFF Press. https://doi.org/10.4324/9781315064215

Duglio, S., \& Beltramo, R. (2017). Estimating the economic impacts of a small-scale sport tourism event: The case of the Italo-Swiss mountain trail CollonTrek. Sustainability, 9(3), 343. https://doi.org/10.3390/ su9030343.

Federación Extremeña de Montaña y Escalada (2020). Archivo de datos de carreras por montaña en Extremadura. http://fexme.com

González, A. X., \& Mundina, J. J. (2014). Experiencia práctica: Actividades físicas y deportivas en el medio natural. Enseñando mediante proyectos en el ámbito universitario. Revista Española de Educación Física 
y Deportes: REEFD, 405, 97-105 Recuperado de http://reefd.es/index. $\mathrm{php} / \mathrm{reefd} / \mathrm{article/viewFile/40/42.}$

González, I. (2019). Evaluación de la calidad percibida de los atletas participantes en un medio maratón internacional. EmásF: Revista Digital de Educación Física, (60), 76-90.https://emasf.webcindario.com/Evaluacion_de_la_calidad_percibida_de_los_atletas_participantes_de_ un_medio_maraton.pdf

González-García, R. J., González-Serrano, M. H., \& Ayora-Pérez, D. (2019). El turismo activo en la comunidad. Relación entre impactos percibidos por los residentes y apoyo al desarrollo del sector. SPORT TK-Revista EuroAmericana De Ciencias Del Deporte, 8(1), 9-16. https:// doi.org/10.6018/sportk.362261

González-Garcia, R.J., Añó, V., Parra-Camacho, D., Calabuig, F. (2018) Perception of residents about the impact of sports tourism on the community: Analysis and scale-validation. J. Phys. Educ. Sport, 18(1) 149-156. https://doi.org/10.7752/jpes.2018.01019

González-Lázaro, J., Frutos de Miguel, J., Arribas-Cubero, H. F.. \& Rodríguez-Marroyo, J. A. (2020) Analysis of the Resilience Scale in Mountain Runners. Revista Internacional de Medicina y Ciencias de la Actividad Física y el Deporte. En prensa. http://cdeporte.rediris.es/revista/inpress/artanalisis1285e.pdf

Guíu, M., \& Leyton, M. (2019). Perfil psicológico en corredores de ultramaratón. Retos, 36, 310-317. https://doi.org/10.47197/retos.v36i36. 69119.

Hair, J. F., Black, W. C., Babin, B. J., Anderson, R. E., \& Tatham, R. L. (2014). Multivariate Data Analysis (7th ed.). Pearson.

Hautbois, C., Djaballah, M., \& Desbordes, M. (2020). The social impact of participative sporting events: A clúster analysis of marathon participants based on perceived benefits. Sport in Society, 23(2), 335-353. https://doi.org/10.1080/17430437.2019.1673371

Hightower, R., Brady, M. K., \& Baker, T. L. (2002). Investigating the role of the physical environment in hedonic serviceconsumption: an exploratory study of sporting events. Journal of Busisness Research, 55(9), 697-707. https://doi.org/10.1016/S0148-2963(00)00211-3

Hyun, M., \& Jordan, J. S. (2020). Athletic goal achievement: A critical antecedent of event satisfaction, re-participation intention, and future exercise intention in participant sport event. Sport Management Review, 23(2), 256-270. doi:10.1016/j.smr.2019.01.007.

Jiménez-Rubio, D. (2015). Sports tourism: trail running as a tool for local development. (Tesis de Máster). Universidad de Alicante.

Karadakis, K. (2012). The Influence of Small-scale Sport Event Impacts on Personal and Community Quality of Life and Support for Sport Event Tourism (Tesis Doctoral). University of Florida.

Kennedy, H., Baker, B. J., Jordan, J. S., \& Funk, D. C. (2019). Running recession: A trend analysis of running involvement and runner charac teristics to understand declining participation. Journal of Sport $\mathrm{Ma}$ nagement, 33(3), 215-228. https://doi.org/10.1123/jsm.2018-0261.

Khalilzadeh, J., \& Tasci, A. D. A. (2017). Large sample size, significance level, and the effect size: Solutions to perils of using big data for academic research. Tourism Management, 62, 89-96. https://doi. org/10.1016/j.tourman.2017.03.026.

Kim, T. H., Ko, Y. J., \& Park, C. M. (2013). The influence of event quality on revisit intention: Gender difference and segmentation strategy. Managing Service Quality, 23(3), 205-224. https://doi. org/10.1108/09604521311312237

Ma, S. C., \& Kaplanidou, K. (2018). Effects of event service quality on the quality of life and behavioral intentions of recreational runners. Leisure Sciences, 1-21. https://doi.org/10.1080/01490400.2018 .1448028 .

Martínez-Cevallos, D., Proaño-Grijalva, A., Alguacil, M., Duclos-Bastías, D., Parra-Camacho, D. (2020). Segmentation of Participants in a Sports Event Using Clúster Analysis. Sustainability, 12(14), 5641. https://doi.org/10.3390/su12145641

Ministerio de Cultura y Deporte (2020). Anuario de estadísticas deportivas 2020. http://www.culturaydeporte.gob.es/dam/jcr:47414879-4f954cae-80c4-e289b3fbced9/anuario-de-estadisticas-deportivas2020.pdf

Moon, K. S., Ko, Y. J., Connaughton, D. P., \& Lee, J. H. (2013). A mediating role of destination image in the relationship between event quality, perceived value, and behavioral intention. Journal of Sport \& Tou rism, 18(1), 49-66. https://doi.org/10.1080/14775085.2013.799960.

Moreno, T. (2014). Análisis de satisfacción de la Gran Trail Aneto-Posets, un recorrido infinito (Trabajo Fin de Máster). Universidad de Zaragoza.

Mur, M., Abella, S. y Barlés, M. J. (2020). Pequeños eventos deportivos y su impacto en el turismo local: el caso de la prueba cicloturista La Quebrantahuesos. Investigaciones Turísticas, 19, 240-264. https://doi. org/10.14198/INTURI2020.19.11

Murtagh, F., \& Legendre, P. (2014). Ward's Hierarchical Agglomerative Clustering Method: Which Algorithms Implement Ward's Criterion? Journal of Classification, 31, 274-295. https://doi.org/10.1007/ s00357-014-9161-z

Ogles, B.M., \& Masters, K.S. (2003). A typology of marathon runners based on cluster analysis of motivations. Journal of Sport Behavior, 26(1), 69-85.

Oñorbe, M. (2014). Diagnóstico de la situación actual de las carreras por montaña en el Parque Nacional de Guadarrama. https://manuelmedioambiente.wordpress.com/2014/07/11/carreras_pnguadarrama/

Parra-Camacho, D., Calabuig-Moreno, F., Añó-Sanz, V., Ayora-Pérez, D., \& Núñez-Pomar, J. M. (2014). El impacto de un evento deportivo mediano: percepción de los residentes de la comunidad de acogida. Retos, 26, 88-93. https://doi.org/10.47197/retos.v0i26.34407.

Parra-Camacho, D., González-Serrano, M. H., González-García, R. J., Calabuig, F. (2019). Sporting Habits of UrbanRunners: Classification According to Their Motivation. International Journal of Environment Research and Public Health, 16(24) 4990. https://doi.org/10.3390/ ijerph16244990

Prieto, J. M. (2016). Razones para correr de corredores populares. Athlos: Revista Internacional de Ciencias Sociales de la Actividad Física, el Juego y el Deporte, 11(7). http://museodeljuego.org/wp-content/uploads/7.Razones-para-correr-de-corredores-populares.pdf

Rejón, F., Alemany, M., \& García, M. A. (2017). Segmentación de los cicloturistas de montaña en las islas baleares a través de las motivaciones de visita: un análisis clúster. En ESIC (ed.) XXIX Congreso Internacional de Marketing AEMARK, (pp 1088-1100). ESIC.

Revelle, W. (2019). psych: Procedures for Psychological, Psychometric, and Personality Research. [R package]. https://cran.r-project.org/ package $=$ psych

Roca-Cruz, A., González-Ruiz, J., Porcel-Rodríguez, P., \& Cabello-Manrique, D. (2017). Impacto económico de los asistentes a la Universiada de Invierno del 2015 en la ciudad de Granada. SPORT TK: Revista Euroamericana de Ciencias del Deporte, 8(1), 7-12. https://doi.org/ https://doi.org/10.6018/sportk.362001.

Romesburg, H. C. (1984). Clúster Analysis for Researchers. Lulu Press.

Ropits Social Running (2016). Runedia. https://runedia.mundodeportivo.com/

Ruiz-Juan, F.; Zarauz, A. (2014). Analysis of motivation amongst Spanish marathon runners. Revista Latinoamericana de Psicología, 46(1), 1-11. https://doi.org/0.1016/S0120-0534(14)70001-9

Sato, M., Jordan, J. S., \& Funk, D. C. (2014). The role of physically active leisure for enhancing quality of life. Leisure Sciences, 36(3), 293-313. https://doi.org/10.1080/01490400.2014.886912.

Scheer, V., Basset, P., Giovanelli, N., Vernillo, G., Millet, G.P., \& Costa, R.J. (2020). Defining off-road running: a position statement from the Ultra Sports Science Foundation. International journal of sports medicine, 41(05), 275-284. https://doi.org/10.1055/a-1096-0980

Seguí, J., \& Farías, E. I. (2018). El trail running (carreras de o por montaña) en España. Inicios, evolución y (actual) estado de la situación. Retos, 33, 123-128. https://doi.org/10.47197/retos.v0i33.56462.

Shonk, D.J., \& Chelladural, P. (2008). Service quality, satisfaction, and intent to return in event sport tourism. Journal of Sport Management, 22(5), 587-602. https://doi.org/10.1123/jsm.22.5.587.

Statista (2020a). Number of participants in trail running in the United States from 2006 to 2017. https://www.statista.com/statistics/191333/participants-in-trail-running-in-the-us-since-2006/.

Statista (2020b). Number of young adult participants in trail running in the United States from 2006 to 2017. https://www.statista.com/ statistics/190948/young-adult-participation-in-trail-running-in-theus-since-2006/.

Theodorakis, N. D., Kaplanidou, K., \& Karabaxoglou, I. (2015). Effect of event service quality and satisfaction on happiness among runners of 
a recurring sport event. Leisure Sciences, 37(1), 87-107. https://doi.or g/10.1080/01490400.2014.938846.

Tkaczynski, A., \& Rundle-Thiele, S. (2020). Event Market Segmentation: A Review Update and Research Agenda. Event Management, 24(2), 277-295. https://doi.org/10.3727/152599519x15506259855913

Tzetzis, G., Alexandris, K., \& Kapsampeli, S. (2014). Predicting visitors' satisfaction and behavioral intentions from service quality in the context of a small-scale outdoor sport event. International Journal of Event and Festival Management, 5(1), 4-21. https://doi.org/10.1108/ IJEFM-04-2013-0006.

Urbański, R. (2018). Trail running-management, organization, safety, evaluation. The activity of ITRA Association. Journal of Education, Health and Sport, 8(8), 1236-1249. http://dx.doi.org/10.5281/zenodo.1468049
Valente, T. I. (2020). Azores Trail Run ${ }^{\circledR}$ as a sustainable vehicle for promotion and local development. Cadernos de Geografia, 41, 97-103. https://doi.org/10.14195/0871-1623_41_7.

Vergara-Ferri, J. M., Angosto, S., \& Parra-Camacho, D. (2020). Efecto de la satisfacción de los residentes entre los impactos percibidos y las intenciones futuras respecto a la celebración de un evento de pequeña escala. Revista iberoamericana de psicología del ejercicio y el deporte, 15(1), 81-91. https://dialnet.unirioja.es/descarga/articulo/7501134.pdf

Ward, J. H. (1963). Hierarchical grouping to optimize an objective function. Journal of the American Statistical Association, 58(301), 236244. https://doi.org/10.1080/01621459.1963.10500845

Zeithaml, V. A., Berry, L. L., \& Parasuraman, A. (1996). The behavioral consequences of service quality. Journal of Marketing, 60(2), 31-46. https://doi.org/10.1177/002224299606000203. 